\title{
Identification of Piecewise Constant Robin Coefficient for the Stokes Problem Using the Levenberg-Marquardt Method
}

\author{
F. KHAYAT ${ }^{1}$
}

\begin{abstract}
In this work, we prove the quadratic convergence of the Levenberg-Marquardt method for the inverse problem of identifying a Robin coefficient for the Stokes system, where we suppose that this parameter is piecewise constant on some non accessible part of the boundary and under the assumption that on this part, the velocity of a given reference solution stays far from zero.
\end{abstract}

keywords : Levenberg-Marquardt method, Robin coefficient, Stokes system, Surrogate functional. MSC(2010): $65 \mathrm{~N} 20 ; 65 \mathrm{~N} 21$.

\section{Introduction}

Levenberg-Marquardt method (LMM) is a Newton type method used to solve nonlinear least squares problems $[3,14,23]$. These problems are encountered when the function is nonlinear with respect to parameters.

More precisely, LMM is a variant of Gauss-Newton (GN) methods for minimizing a least square cost $\frac{1}{2}\|g(x)\|^{2}$. The pure form of GN method consists on taking in each iteration : $x^{k+1}=x^{k}+d^{k}$ where the descent direction is

$$
d^{k}=-\left[\nabla g\left(x^{k}\right) \nabla g\left(x^{k}\right)^{\prime}\right]^{-1} \nabla g\left(x^{k}\right) g\left(x^{k}\right) .
$$

To avoid the case when the matrix $\nabla g\left(x^{k}\right) \nabla g\left(x^{k}\right)^{\prime}$ is singular, the GN method is often implemented in a modified form where the latest matrix is replaced by the positive definite one : $\nabla g\left(x^{k}\right) \nabla g\left(x^{k}\right)^{\prime}+\Delta^{k}$. For the LM method, $\Delta^{k}$ is chosen as a positive multiple of the identity matrix.

This method was widely used $[16,19,20,29,30]$ because of its simplicity and because it's known to have a quadratic rate of convergence under nonsingularity condition. In [29], the authors have shown, when solving a system of nonlinear equations $F(x)=0$, that the rate of convergence still quadratic under less stringent assumption that is $\|F(x)\|$ provides a local error bound. For this doing they have taken $\Delta^{k}=\mu_{k} I$, with $\mu_{k}=\left\|F\left(x^{k}\right)\right\|^{2}$. In the present work, we want to use the LM method and the latest study in order to solve the inverse problem consisting on the reconstruction of the Robin coefficient on some non accessible part of the boundary of a domain governed by the Stokes system from measurements available on some accessible part. This problem is well known to be ill-posed and instable due to the presence of noise in the observed data. The problem is often formulated as a least-square nonlinear and non-convex minimization with a Tikhonov regularization term.

Such problem can model for example the blood flow in cardiovascular system [4, 18, 27]. In [18], the authors was interested by the study of a medical device called the stent and used to prevent rupture of cerebral aneurysms. The blood flow was modelized by Navier-Stokes equations and the stent by a porous media with a dissipative term.

Such kind of problem was also considered in the study of the airflow in the lungs $[1,24]$. Indeed, the airway resistance, modeled by a positive constante, characterizes in pneumology the patient ventilation capability. It quantifies the relationship between the airflow rate through the respiratory tract and the pressure drop between its ends.

Many works have delt with the problem of identifying a Robin type coefficient. Among them, let us cite in a non-exhaustive way, the works of Chaabane et al. [6] or those of Inglese et al [17] where the authors have considered the case of the Laplace equation. In $[4,5]$, the authors have focused on the question of the identifiability and have established some stability results for such kind of inverse problems corresponding to Stokes and

\footnotetext{
${ }^{1}$ Adress: LAMSIN-ENIT-BP 37, 1002 Tunis le Belvédère, Tunisia. e-mail: faten.khayat@gmail.com
} 
Navier-Stokes systems. A brieve numerical study based on genetic algorithm was presented in [11].

Several numerical methods have been used for identifying the Robin coefficient. Among them, the GaussNewton method with no regularization was used in [15], the conjugate gradient method was applied in [21]. An L1-tracking functional approach was proposed in [7] for the Laplace equation while a gradient type method for a piecewise constant Robin parameter was treated in [8]. A modified conjugate gradient method has been used in [28]. The use of LM method for identifying some Robin coefficient can be found in [31] were this method was applied for both a scalar elliptic problem and a time-dependent parabolic system. In [9], the authors have investigated a simultaneous reconstruction of Robin coefficient and heat flux for the heat equation. In [20], a parameter identification in inverse groundwater hydrology was done using the LM scheme.

All of these studies was concerned either by the Laplace or by the diffusion equation. The case of Stokes system was studied in [2] where the authors have considered partially overspecified data on the accessible part of the boundary and supposed that the Robin coefficient is constant. Up to our knowledge, no previous work has been devoted to the case that we consider here.

The paper is organized as follows. In section 2 we give some definitions and some preliminary results. In section 3, we present the Levenberg-Marquardt method and prove its quadratic convergence for the reconstruction of the Robin coefficient under some corresponding assumptions. In section 4, we introduce the surrogate functional technique in order to simplify the numerical minimization procedure. Section 5 is reserved to some numerical results. We finish by some concluding remarks.

\section{Preliminary results}

Let $\Omega \subset \mathbb{R}^{d}$, for $d=2,3$, be an open Lipschitz bounded and connected domain such that $\partial \Omega=\Gamma_{\ell} \cup \Gamma_{0} \cup \Gamma_{\text {out }}$ and suppose that $\Gamma_{\text {out }}=\cup_{i=1}^{N} \Gamma_{i}$ where $N \in \mathbb{N}$. Consider the following Stokes problem with mixed Dirichlet, Neumann and Robin boundary conditions, governing the flow of an incompressible fluid:

$$
\begin{cases}-\Delta u+\nabla p=0 & \text { in } \Omega \\ \nabla \cdot u=0 & \text { in } \Omega \\ u=0 & \text { on } \Gamma_{\ell} \\ \frac{\partial u}{\partial n}-p n=g & \text { on } \Gamma_{0} \\ \frac{\partial u}{\partial n}-p n+R u=0 & \text { on } \Gamma_{\text {out }}\end{cases}
$$

$u$ is the velocity of the fluid, $p$ is the pressure and $R$ is the Robin coefficient. In the sequel, we will use the following notations: for $\Gamma$ subset of the boundary $\partial \Omega, H^{1 / 2}(\Gamma)$ is the space of the traces over $\Gamma$ of elements of $H^{1}(\Omega)$ and $H^{-1 / 2}(\Gamma)$ is its topological dual space. $L_{0}^{2}(\Omega)=\left\{q \in L^{2}(\Omega): \int_{\Omega} q=0\right\}$. We use the symbol $\langle;\rangle_{L^{2}(\Gamma)}$ for the inner product in $L^{2}(\Gamma)$ and $\|\cdot\|_{L^{2}(\Gamma)}$ for its norm. We suppose $g \in H^{-1 / 2}\left(\Gamma_{0}\right)$. We also define the space:

$$
V=\left\{v \in H^{1}(\Omega) ; \operatorname{div} v=0 \text { and } v_{\mid \Gamma_{\ell}}=0\right\}
$$

and the set:

$$
\Gamma^{i n}=\{x \in \Gamma / d(x, \partial \overline{\Omega \backslash \Gamma})>0\} .
$$

We will assume for the sequel that:

$$
R_{\mid \Gamma_{i}}=R_{i} \in \mathbb{R}_{+} \text {on } \Gamma_{i}, \forall i=1,2, \cdots, N .
$$

Define the set of admissible coefficients:

$$
K=\left\{R \in L^{\infty}\left(\Gamma_{\text {out }}\right) / 0<r_{1} \leq R(x) \leq r_{2} \text { a.e. on } \Gamma_{\text {out }}\right\},
$$


where $r_{1}$ and $r_{2}$ are two positive constants.

We are concerned in this work with the inverse problem of identifying $R$ on $\Gamma_{\text {out }}$ from measurements available on $\Gamma_{0}$ : Given $u$ and $p$ on $\Gamma_{0}$,

$$
\begin{aligned}
& \text { find } R \in K \text { s.t. }(u, p) \in H^{1}(\Omega)^{2} \times L_{0}^{2}(\Omega) \text { is the solution of } \\
& \begin{cases}-\Delta u+\nabla p=0 & \text { in } \Omega \\
\nabla \cdot u=0 & \text { in } \Omega \\
u=0 & \text { on } \Gamma_{\ell} \\
\frac{\partial u}{\partial n}-p n=g & \text { on } \Gamma_{0} \\
\frac{\partial u}{\partial n}-p n+R u=0 & \text { on } \Gamma_{\text {out }}\end{cases}
\end{aligned}
$$

We must begin by recalling essential results concerning the well-posedness and the regularity of the forward problem also with the uniqueness and the stability of the inverse one. These results are stated in $[11,12,13]$ :

Proposition 1 (Global Regularity)

Let $R_{M}>0,1_{\Gamma_{0}} g \in H^{-1 / 2}(\partial \Omega)$ and assume that $R$ satisfies (3). Then system (1) admits a unique solution $(u, p) \in V \times L^{2}(\Omega)$. Moreover, if we assume that $R \leq R_{M}$, there exists $C\left(R_{M}\right)>0$ such that:

$$
\|u\|_{H^{1}(\Omega)}+\|p\|_{L^{2}(\Omega)} \leq C\left(R_{M}\right)\left\|1_{\Gamma_{0}} g\right\|_{H^{-1 / 2}(\partial \Omega)}
$$

Proposition 2 (Local Regularity)

Let $m \in \mathbb{N}^{\star}, R_{M}>0$ and $x_{0} \in \Gamma_{0}^{i n}$. We assume that $\Gamma_{0}$ is of class $C^{m, 1}$ if $m \geq 1$ and Lipschitz otherwise. Let $1_{\Gamma_{0}} g \in H^{m-\frac{1}{2}}(\partial \Omega)$ and assume that $R$ satisfies (3). Then there exists $\rho>0$ such that the solution $(u, p)$ of problem (1) belongs to $H^{m+1}\left(B_{\rho}\left(x_{0}\right) \cap \Omega\right) \times H^{m}\left(B_{\rho}\left(x_{0}\right) \cap \Omega\right)$. Furthermore, if we assume that $R \leq R_{M}$, there exists a constant $C\left(R_{M}\right)>0$ such that:

$$
\|u\|_{H^{m+1}\left(B_{\rho}\left(x_{0}\right) \cap \Omega\right)}+\|p\|_{H^{m}\left(B_{\rho}\left(x_{0}\right) \cap \Omega\right)} \leq C\left(R_{M}\right)\left\|1_{\Gamma_{0} g}\right\|_{H^{m-\frac{1}{2}}(\partial \Omega)}
$$

Proposition 3 (Uniqueness)

Let $x_{0} \in \Gamma_{0}$ and $r>0$. Suppose that $g$ is non identically zero on $\Gamma_{0}$. Let $\left(u_{k}, p_{k}\right) \in H^{1}(\Omega) \times L^{2}(\Omega)$ be a solution of the problem (1) with $R=R^{k}$, for $k=1,2$.

If $u_{1}=u_{2}$ on $B\left(x_{0}, r\right) \cap \Gamma_{0}$ then $R^{1}=R^{2}$ on $\Gamma_{\text {out }}$.

Theorem 4 (Stability)

Assume that $\Gamma_{0}$ is of class $C^{\infty}$ and $\Gamma_{i}$ is of class $C^{2,1}$ for $i=1,2, \cdots, N$. Let $m>0, R_{M}>0, M_{1}>0$ and $\Gamma \subseteq \Gamma_{0}$ be a non empty open subset of $\partial \Omega$ such that $\left(\bar{\Gamma} \cap \bar{\Gamma}_{\ell}\right) \cup\left(\bar{\Gamma} \cap \bar{\Gamma}_{\text {out }}\right)=\varnothing$ and let $g \in H^{\frac{3}{2}}\left(\Gamma_{0}\right)$ be non identically zero on $\Gamma_{0}$ and such that $\|g\|_{H^{\frac{3}{2}}\left(\Gamma_{0}\right)} \leq M_{1}$. We assume that $R^{k}$ satisfies (3) with $R_{i}=R_{i}^{k}$ be such that $R_{i}^{k} \leq R_{M}$ for $i=1,2, \cdots, N$ and for $k=1,2$. Let us denote by $\left(u_{k}, p_{k}\right)$ the solution of (1) associated to $R=R^{k}$ for $k=1,2$. We assume that there exists $x_{j} \in \Gamma_{j}^{i n}$ such that $\left|u_{2}\left(x_{j}\right)\right|>m$ for all $j=1,2, \cdots, N$.

Then, there exists $C_{s}=C_{s}\left(R_{M}, M_{1}, N, m\right)>0$ such that

$$
\left\|R^{1}-R^{2}\right\|_{L^{\infty}\left(\Gamma_{\text {out }}\right)} \leq C_{s}\left(\left\|u_{1}-u_{2}\right\|_{L^{2}(\Gamma)}+\left\|p_{1}-p_{2}\right\|_{L^{2}(\Gamma)}+\left\|\frac{\partial p_{1}}{\partial n}-\frac{\partial p_{2}}{\partial n}\right\|_{L^{2}(\Gamma)}\right)
$$

\section{Remark 5}

If the Robin coefficient is not piecewise constant, the stability results established in [4, 5] are of logarithmic type and so we can't hope prove the quadratic convergence of the LM method in that case.

Let $R^{\star}$ be the exact solution of (5) and $z^{\delta}$ be the observed noisy data with respect to the true solution $u\left(R^{\star}\right), \delta$ is the noise level. Then we have:

$$
\left\|u\left(R^{\star}\right)-z^{\delta}\right\|_{L^{2}\left(\Gamma_{0}\right)} \leq \delta
$$


We will assume throughout this work that:

$$
u\left(R^{\star}\right) \in L^{\infty}\left(\Gamma_{\text {out }}\right)
$$

The inverse problem of identifying the Robin coefficient $\mathrm{R}$ is formulated into the following stabilized minimization:

$$
\min _{R \in K} \mathcal{J}(R) ; \mathcal{J}(R)=\left\|u(R)-z^{\delta}\right\|_{L^{2}\left(\Gamma_{0}\right)}^{2}+\beta\left\|R-R^{\star}\right\|_{L^{2}\left(\Gamma_{\text {out }}\right)}^{2}
$$

where $\left\|R-R^{\star}\right\|_{L^{2}\left(\Gamma_{\text {out }}\right)}^{2}$ is the Tikhonov regularization term and $\beta$ is the regularization parameter.

For the following study, we need to define the couple $(w, s)=\left(u^{\prime}(R) d, p^{\prime}(R) d\right)$ the Fréchet derivative at direction $d$ of $(u(R), p(R))$ the forward solution of (1). It's easy to verify that $(w, s)$ is solution of the problem:

$$
\begin{cases}-\Delta w+\nabla s=0 & \text { in } \Omega \\ \nabla \cdot w=0 & \text { in } \Omega \\ w=0 & \text { on } \Gamma_{\ell} \\ \frac{\partial w}{\partial n}-s n=0 & \text { on } \Gamma_{0} \\ \frac{\partial w}{\partial n}-s n+R w=-d u(R) & \text { on } \Gamma_{\text {out }}\end{cases}
$$

Let $u^{\prime}(R)^{\star}$ and $p^{\prime}(R)^{\star}$ be the adjoint operators of $u^{\prime}(R)$ and $p^{\prime}(R)$ respectively, and let us note $\left(w^{\star}, s^{\star}\right)=$ $\left(u^{\prime}(R)^{\star} h, p^{\prime}(R)^{\star} h\right)$ at a direction $h$. Then $\left(w^{\star}, s^{\star}\right)$ is solution of the problem:

$$
\begin{cases}-\Delta w^{\star}+\nabla s^{\star}=0 & \text { in } \Omega \\ \nabla \cdot w^{\star}=0 & \text { in } \Omega \\ w^{\star}=0 & \text { on } \Gamma_{\ell} \\ \frac{\partial w^{\star}}{\partial n}-s^{\star} n=-h u(R) & \text { on } \Gamma_{0} \\ \frac{\partial w^{\star}}{\partial n}-s^{\star} n+R w^{\star}=0 & \text { on } \Gamma_{\text {out }}\end{cases}
$$

The relation between $w$ and $w^{\star}$ is then given by:

\section{Lemma 6}

For any directions $d$ and $h$, we have the following relation:

$$
\left\langle u^{\prime}(R) d ; u(R) h\right\rangle_{L^{2}\left(\Gamma_{0}\right)}=\left\langle u(R) d ; u^{\prime}(R)^{\star} h\right\rangle_{L^{2}\left(\Gamma_{\text {out }}\right)} .
$$

Proof

The variational formulation of (10) is, for all $\varphi \in V$ :

$$
\int_{\Omega} \nabla w: \nabla \varphi-\int_{\partial \Omega}\left[\frac{\partial w}{\partial n}-s n\right] \varphi=0
$$

using the limit condition of $\varphi$ on $\Gamma_{\ell}$ and of $w$ on $\Gamma_{0}$, we obtain:

$$
\int_{\Omega} \nabla w: \nabla \varphi+\int_{\Gamma_{\text {out }}} R w \varphi=-\int_{\Gamma_{\text {out }}} d u(R) \varphi
$$

In the same manner, the variational formulation of (11) is given, for all $\psi \in V$, by:

$$
\int_{\Omega} \nabla w^{\star}: \nabla \psi+\int_{\Gamma_{\text {out }}} R w^{\star} \psi=-\int_{\Gamma_{0}} h u(R) \psi
$$


Taking $\varphi=w^{\star}$ in (13) and $\psi=w$ in (14), we obtain:

$$
\int_{\Gamma_{0}} h u(R) w=\int_{\Gamma_{\text {out }}} d u(R) w^{\star}
$$

\section{The Levenberg-Marquardt Method}

First, let us recall the definition of Levenberg-Marquardt method given in [29]. Consider, for $F: \mathbb{R}^{n} \longrightarrow \mathbb{R}^{m}$ a continuously differentiable function, the system of nonlinear equations :

$$
F(x)=0
$$

and suppose that (15) has a nonempty solution set $X^{\star}$.

\section{Definition 7}

The iterate $x^{k+1}$ by the Levenberg-Marquardt Method (LMM) is defined by:

$$
x^{k+1}=x^{k}+d^{k}
$$

where $d^{k}$ is the solution of the linear system:

$$
\left[F^{\prime}\left(x^{k}\right)^{T} F^{\prime}\left(x^{k}\right)+\mu_{k} I\right] d=-F^{\prime}\left(x^{k}\right)^{T} F\left(x^{k}\right)
$$

which is equivalent to the unconstrained minimization problem :

$$
\min _{d \in \mathbb{R}^{n}} \theta^{k}
$$

where $\theta^{k}: \mathbb{R}^{n} \longrightarrow \mathbb{R}$ is a strictly convex function defined by :

$$
\theta^{k}(d)=\left\|F^{\prime}\left(x^{k}\right) d+F\left(x^{k}\right)\right\|^{2}+\mu_{k}\|d\|^{2} .
$$

The minimization problem (9) is nonlinear and non-convex with respect to the Robin coefficient $R$ because of the nonlinearity of the forward solution $u(R)$ with respect to $R$. To change $(9)$ into a convex minimization we apply the LM method. So we begin by applying the following linearization, for a given $\bar{R} \in K[9,20,31]$ :

$$
u(R) \approx u(\bar{R})+u^{\prime}(\bar{R})(R-\bar{R})
$$

Replacing in (9), we obtain the Levenberg-Marquardt iteration:

$$
R^{k+1}=\underset{R \in K}{\operatorname{argmin}} \mathcal{J}(R)=\underset{R \in K}{\arg \min }\left\{\left\|u^{\prime}\left(R^{k}\right)\left(R-R^{k}\right)-\left(z^{\delta}-u\left(R^{k}\right)\right)\right\|_{L^{2}\left(\Gamma_{0}\right)}^{2}+\beta_{k}\left\|R-R^{k}\right\|_{L^{2}\left(\Gamma_{\text {out }}\right)}^{2}\right\}
$$

\section{Lemma 8}

Assume that for $R \in K, u(R) \in L^{\infty}\left(\Gamma_{\text {out }}\right)$, then there exists $L>0$ and $c_{1}>0$ such that for all $R, \bar{R} \in K$ and for any direction $d$ :

$$
\begin{gathered}
\|u(R)-u(\bar{R})\|_{L^{2}\left(\Gamma_{0}\right)} \leq L\|R-\bar{R}\|_{L^{2}\left(\Gamma_{\text {out }}\right)} \\
\left\|u^{\prime}(R) d\right\|_{L^{2}(\partial \Omega)} \leq L\|d\|_{L^{2}\left(\Gamma_{\text {out }}\right)} \\
\left\|u^{\prime}(\bar{R})(R-\bar{R})-(u(R)-u(\bar{R}))\right\|_{L^{2}\left(\Gamma_{0}\right)} \leq c_{1}\|R-\bar{R}\|_{L^{2}\left(\Gamma_{\text {out }}\right)}^{2}
\end{gathered}
$$


Proof

The variationnal form of problem (1) is given, for all $\varphi \in V$, by:

$$
\int_{\Omega} \nabla u(R): \nabla \varphi+\int_{\Gamma_{\text {out }}} R u(R) \varphi=\int_{\Gamma_{0}} g \varphi .
$$

In the same manner, we have:

$$
\int_{\Omega} \nabla u(\bar{R}): \nabla \varphi+\int_{\Gamma_{\text {out }}} \bar{R} u(\bar{R}) \varphi=\int_{\Gamma_{0}} g \varphi .
$$

equalizing (24) and (25), we obtain:

$$
\int_{\Omega} \nabla[u(R)-u(\bar{R})]: \nabla \varphi=\int_{\Gamma_{\text {out }}}[-R u(R)+\bar{R} u(\bar{R})] \varphi
$$

or equivalently:

$$
\int_{\Omega} \nabla[u(R)-u(\bar{R})]: \nabla \varphi+\int_{\Gamma_{\text {out }}} \bar{R}[u(R)-u(\bar{R})] \varphi=\int_{\Gamma_{\text {out }}}(\bar{R}-R) u(R) \varphi .
$$

Taking $\varphi=u(R)-u(\bar{R})$, we obtain:

$$
\int_{\Omega}|\nabla[u(R)-u(\bar{R})]|^{2}+\int_{\Gamma_{\text {out }}} \bar{R}|u(R)-u(\bar{R})|^{2}=\int_{\Gamma_{\text {out }}}(\bar{R}-R) u(R)[u(R)-u(\bar{R})]
$$

using Korn's inequality [25], we can assert that there exists a constant $c_{k r}>0$ such that:

$$
\begin{aligned}
c_{k r}\|u(R)-u(\bar{R})\|_{1, \Omega}^{2}+r_{1}\|u(R)-u(\bar{R})\|_{L^{2}\left(\Gamma_{\text {out }}\right)}^{2} & \leq\|u(R)\|_{L^{\infty}\left(\Gamma_{\text {out }}\right)}\|R-\bar{R}\|_{L^{2}\left(\Gamma_{\text {out }}\right)}\|u(R)-u(\bar{R})\|_{L^{2}\left(\Gamma_{\text {out }}\right)} \\
& \leq c_{2}\|R-\bar{R}\|_{L^{2}\left(\Gamma_{\text {out }}\right)}\|u(R)-u(\bar{R})\|_{L^{2}\left(\Gamma_{\text {out }}\right)}
\end{aligned}
$$

where $c_{2}=\|u(R)\|_{L^{\infty}\left(\Gamma_{\text {out }}\right)}$ and $r_{1}$ given in (4). We deduce consecutively:

$$
\|u(R)-u(\bar{R})\|_{L^{2}\left(\Gamma_{\text {out }}\right)} \leq \frac{c_{2}}{r_{1}}\|R-\bar{R}\|_{L^{2}\left(\Gamma_{\text {out }}\right)}
$$

and:

$$
c_{k r}\|u(R)-u(\bar{R})\|_{1, \Omega}^{2} \leq c_{2}\|R-\bar{R}\|_{L^{2}\left(\Gamma_{\text {out }}\right)}\|u(R)-u(\bar{R})\|_{L^{2}\left(\Gamma_{\text {out }}\right)}
$$

and consequently that:

$$
\|u(R)-u(\bar{R})\|_{1, \Omega}^{2} \leq \frac{c_{2}^{2}}{r_{1} c_{k r}}\|R-\bar{R}\|_{L^{2}\left(\Gamma_{\text {out }}\right)}^{2}
$$

which implies (21) by applying the trace theorem and setting $L=\frac{c_{2}}{\sqrt{r_{1} c_{k r}}}$. To prove (22), we take in (13) $\varphi=w=u^{\prime}(R) d$. We then obtain:

$$
\int_{\Omega}|\nabla w|^{2}+\int_{\Gamma_{\text {out }}} R|w|^{2}=\int_{\Gamma_{\text {out }}}-d u(R) w
$$

which implies:

$$
r_{1} \int_{\Gamma_{\text {out }}}|w|^{2} \leq c_{2}\|d\|_{L^{2}\left(\Gamma_{\text {out }}\right)}\|w\|_{L^{2}\left(\Gamma_{\text {out }}\right)}
$$


This last inequality gives:

$$
\|w\|_{L^{2}\left(\Gamma_{\text {out }}\right)} \leq \frac{c_{2}}{r_{1}}\|d\|_{L^{2}\left(\Gamma_{\text {out }}\right)} .
$$

On the other hand, we can deduce from (28) that:

$$
c_{k r}\|w\|_{1, \Omega}^{2} \leq c_{2}\|d\|_{L^{2}\left(\Gamma_{\text {out }}\right)}\|w\|_{L^{2}\left(\Gamma_{\text {out }}\right)}
$$

which gives, using (29):

$$
c_{k r}\left\|u^{\prime}(R) d\right\|_{1, \Omega}^{2} \leq \frac{c_{2}^{2}}{r_{1}}\|d\|_{L^{2}\left(\Gamma_{\text {out }}\right)}^{2}
$$

or equivalently, by applying the trace theorem:

$$
\left\|u^{\prime}(R) d\right\|_{L^{2}(\partial \Omega)} \leq L\|d\|_{L^{2}\left(\Gamma_{\text {out }}\right)} .
$$

Next, we take in (13), $R=\bar{R}$ and $d=R-\bar{R}$, we obtain:

$$
\int_{\Omega} \nabla\left[u^{\prime}(\bar{R})(R-\bar{R})\right]: \nabla \varphi+\int_{\Gamma_{\text {out }}} \bar{R} u^{\prime}(\bar{R})(R-\bar{R}) \varphi=-\int_{\Gamma_{\text {out }}}(R-\bar{R}) u(\bar{R}) \varphi, \quad \forall \varphi \in V .
$$

Substracting (26) from (31) and taking $\varphi=u^{\prime}(\bar{R})(R-\bar{R})-[u(R)-u(\bar{R})]$ gives:

$$
\begin{aligned}
\int_{\Omega} \mid \nabla\left[u^{\prime}(\bar{R})(R-\bar{R})\right. & -(u(R)-u(\bar{R}))]\left.\right|^{2}+\int_{\Gamma_{\text {out }}} \bar{R}\left|u^{\prime}(\bar{R})(R-\bar{R})-(u(R)-u(\bar{R}))\right|^{2} \\
& =\int_{\Gamma_{\text {out }}}(R-\bar{R})(u(R)-u(\bar{R}))\left[u^{\prime}(\bar{R})(R-\bar{R})-(u(R)-u(\bar{R}))\right]
\end{aligned}
$$

thus, there exists $c_{3}>0$ such that:

$$
\begin{aligned}
& \left\|u^{\prime}(\bar{R})(R-\bar{R})-(u(R)-u(\bar{R}))\right\|_{1, \Omega}^{2} \\
& \quad \leq c_{3}\|(R-\bar{R})(u(R)-u(\bar{R}))\|_{L^{2}\left(\Gamma_{\text {out }}\right)}\left\|u^{\prime}(\bar{R})(R-\bar{R})-(u(R)-u(\bar{R}))\right\|_{L^{2}\left(\Gamma_{\text {out }}\right)}
\end{aligned}
$$

using trace theorem, we obtain:

$$
\left\|u^{\prime}(\bar{R})(R-\bar{R})-(u(R)-u(\bar{R}))\right\|_{L^{2}\left(\Gamma_{\text {out }}\right)} \leq c_{3}\|(R-\bar{R})(u(R)-u(\bar{R}))\|_{L^{2}\left(\Gamma_{\text {out }}\right)} .
$$

By the mean value theorem, we can write $u(R)-u(\bar{R})=u^{\prime}(\xi)(R-\bar{R})$ for some $\xi \in K$ and thus using (22), we can assert that there exists a positive constant $c_{4}$ such that:

$$
\left\|u^{\prime}(\bar{R})(R-\bar{R})-(u(R)-u(\bar{R}))\right\|_{L^{2}\left(\Gamma_{\text {out }}\right)} \leq c_{4}\|(R-\bar{R})\|_{L^{2}\left(\Gamma_{\text {out }}\right)}^{2} .
$$

Using (34) in (32), gives:

$$
\left\|u^{\prime}(\bar{R})(R-\bar{R})-(u(R)-u(\bar{R}))\right\|_{1, \Omega}^{2} \leq c_{3} c_{4}\|(R-\bar{R})(u(R)-u(\bar{R}))\|_{L^{2}\left(\Gamma_{\text {out }}\right)}\|(R-\bar{R})\|_{L^{2}\left(\Gamma_{\text {out }}\right)}^{2}
$$

which implies, when using again the mean value theorem and (22), that there exists $c_{1}>0$ such that:

$$
\left\|u^{\prime}(\bar{R})(R-\bar{R})-(u(R)-u(\bar{R}))\right\|_{L^{2}\left(\Gamma_{0}\right)}^{2} \leq c_{1}^{2}\|(R-\bar{R})\|_{L^{2}\left(\Gamma_{\text {out }}\right)}^{4} .
$$

To prove the convergence of the LM method, we assume for the sequel that the hypothesis of Theorem 4 hold true and that there exists $b \in(0,1)$ and a constant $c_{5}=c_{5}(b)>0$ such that for all $R \in N\left(R^{\star}, b\right)$ :

$$
c_{5}\left\|R-R^{\star}\right\|_{L^{\infty}\left(\Gamma_{\text {out }}\right)} \leq\left\|u(R)-u\left(R^{\star}\right)\right\|_{L^{2}(\Gamma)}+\left\|p(R)-p\left(R^{\star}\right)\right\|_{L^{2}(\Gamma)}+\left\|\frac{\partial p(R)}{\partial n}-\frac{\partial p\left(R^{\star}\right)}{\partial n}\right\|_{L^{2}(\Gamma)}
$$


where we have set:

$$
N\left(R^{\star}, b\right)=\left\{R \in K /\left\|R-R^{\star}\right\|_{L^{2}\left(\Gamma_{\text {out }}\right)} \leq b\right\} .
$$

We will also make the following assumption:

There exists a positve constant $C_{M}$ such that $\forall R, \bar{R} \in N\left(R^{\star}, b\right)$ :

$$
\|p(R)-p(\bar{R})\|_{L^{2}(\Gamma)} \quad+\left\|\frac{\partial p(R)}{\partial n}-\frac{\partial p(\bar{R})}{\partial n}\right\|_{L^{2}(\Gamma)} \leq C_{M}\|u(R)-u(\bar{R})\|_{L^{2}(\Gamma)}
$$

This condition combined with (35) gives, for all $R \in N\left(R^{\star}, b\right)$ :

$$
c_{5}\left\|R-R^{\star}\right\|_{L^{\infty}\left(\Gamma_{\text {out }}\right)} \leq\left(1+C_{M}\right)\left\|u(R)-u\left(R^{\star}\right)\right\|_{L^{2}(\Gamma)} .
$$

\section{Remark 9}

1. In (35), we can suppose without loss of generality that $c_{5}<1$.

2. The assumption (35) holds naturally when the hypothesis of the stability theorem (Theorem 4) are fulfilled.

3. Assumption (37) is not a restrictive one having regard to the result of Proposition 2.

To maximize the rate of convergence, we will take at the $k^{\text {th }}$ iteration of the LM method (see $\left.[16,29,31]\right)$ :

$$
\beta_{k}=\left\|u\left(R^{k}\right)-z^{\delta}\right\|_{L^{2}\left(\Gamma_{0}\right)}^{2} .
$$

We will consider the following stopping criterion:

$$
\left\|R^{k}-R^{\star}\right\|_{L^{2}\left(\Gamma_{\text {out }}\right)} \leq 2 \delta \text { or }\left\|u\left(R^{k}\right)-z^{\delta}\right\|_{L^{2}\left(\Gamma_{0}\right)} \leq \sqrt{\delta}
$$

We can now prove the following Lemma:

Lemma 10

Under the hypothesis (8), (35), (37), (39) and (40), if $R^{k} \in N\left(R^{\star}, b\right)$ then $R^{k+1}$ generated by (20) verifies:

$$
\begin{gathered}
\left\|u^{\prime}\left(R^{k}\right)\left(R^{k+1}-R^{k}\right)-\left(z^{\delta}-u\left(R^{k}\right)\right)\right\|_{L^{2}\left(\Gamma_{0}\right)} \leq c_{6}\left(\left\|R^{k}-R^{\star}\right\|_{L^{2}\left(\Gamma_{\text {out }}\right)}^{2}+\delta\right) \\
\left\|R^{k+1}-R^{k}\right\|_{L^{2}\left(\Gamma_{\text {out }}\right)}^{2} \leq c_{7}\left(\left\|R^{k}-R^{\star}\right\|_{L^{2}\left(\Gamma_{\text {out }}\right)}^{2}+\delta\right)
\end{gathered}
$$

for $\delta$ sufficiently small and where $c_{6}=\sqrt{3} \sqrt{\max \left\{c_{1}^{2}+L^{2} ; 1\right\}}$ and $c_{7}=\max \left\{1+2 c_{1}^{2} \delta^{-1} b^{2} ; 2\right\}, L$ and $c_{1}$ are defined in Lemma 8.

Proof

We have

$$
\begin{gathered}
\mathcal{J}\left(R^{\star}\right)=\left\|u^{\prime}\left(R^{k}\right)\left(R^{\star}-R^{k}\right)-\left(z^{\delta}-u\left(R^{k}\right)\right)\right\|_{L^{2}\left(\Gamma_{0}\right)}^{2}+\beta_{k}\left\|R^{\star}-R^{k}\right\|_{L^{2}\left(\Gamma_{\text {out }}\right)}^{2} \\
\leq 2\left\|u^{\prime}\left(R^{k}\right)\left(R^{\star}-R^{k}\right)-\left(u\left(R^{\star}\right)-u\left(R^{k}\right)\right)\right\|_{L^{2}\left(\Gamma_{0}\right)}^{2}+2\left\|u\left(R^{\star}\right)-z^{\delta}\right\|_{L^{2}\left(\Gamma_{0}\right)}^{2} \\
+\beta_{k}\left\|R^{\star}-R^{k}\right\|_{L^{2}\left(\Gamma_{\text {out }}\right)}^{2}
\end{gathered}
$$

using (7 ) and (23), we obtain:

$$
\mathcal{J}\left(R^{\star}\right) \leq 2 c_{1}^{2}\left\|R^{\star}-R^{k}\right\|_{L^{2}\left(\Gamma_{\text {out }}\right)}^{4}+2 \delta^{2}+\beta_{k}\left\|R^{\star}-R^{k}\right\|_{L^{2}\left(\Gamma_{\text {out }}\right)}^{2} .
$$

Using (21), we can write

$$
\begin{aligned}
\beta_{k}=\left\|u\left(R^{k}\right)-z^{\delta}\right\|_{L^{2}\left(\Gamma_{0}\right)}^{2} & \leq 2\left\|u\left(R^{k}\right)-u\left(R^{\star}\right)\right\|_{L^{2}\left(\Gamma_{0}\right)}^{2}+2\left\|u\left(R^{\star}\right)-z^{\delta}\right\|_{L^{2}\left(\Gamma_{0}\right)}^{2} \\
& \leq 2 L^{2}\left\|R^{k}-R^{\star}\right\|_{L^{2}\left(\Gamma_{\text {out }}\right)}^{2}+2 \delta^{2}
\end{aligned}
$$


we then have the majoration:

$$
\begin{aligned}
\mathcal{J}\left(R^{\star}\right) & \leq 2 c_{1}^{2}\left\|R^{\star}-R^{k}\right\|_{L^{2}\left(\Gamma_{\text {out }}\right)}^{4}+2 \delta^{2}+\left[2 L^{2}\left\|R^{k}-R^{\star}\right\|_{L^{2}\left(\Gamma_{\text {out }}\right)}^{2}+2 \delta^{2}\right]\left\|R^{\star}-R^{k}\right\|_{L^{2}\left(\Gamma_{\text {out }}\right)}^{2} \\
& \leq\left(2 c_{1}^{2}+2 L^{2}\right)\left\|R^{\star}-R^{k}\right\|_{L^{2}\left(\Gamma_{\text {out }}\right)}^{4}+2 \delta^{2}+2 \delta^{2}\left\|R^{\star}-R^{k}\right\|_{L^{2}\left(\Gamma_{\text {out }}\right)}^{2} .
\end{aligned}
$$

So, for $\delta \leq 2 \sqrt{c_{1}^{2}+L^{2}}$, it's easy to verify that:

$$
\mathcal{J}\left(R^{\star}\right) \leq 2\left(\sqrt{c_{1}^{2}+L^{2}}\left\|R^{\star}-R^{k}\right\|_{L^{2}\left(\Gamma_{\text {out }}\right)}^{2}+\delta\right)^{2}
$$

Since $\mathcal{J}\left(R^{k+1}\right) \leq \mathcal{J}\left(R^{\star}\right)$ and $\left\|u^{\prime}\left(R^{k}\right)\left(R^{k+1}-R^{k}\right)-\left(z^{\delta}-u\left(R^{k}\right)\right)\right\|_{L^{2}\left(\Gamma_{0}\right)}^{2} \leq \mathcal{J}\left(R^{k+1}\right)$, we deduce:

$$
\left\|u^{\prime}\left(R^{k}\right)\left(R^{k+1}-R^{k}\right)-\left(z^{\delta}-u\left(R^{k}\right)\right)\right\|_{L^{2}\left(\Gamma_{0}\right)}^{2} \leq 2 \max \left\{c_{1}^{2}+L^{2} ; 1\right\}\left(\left\|R^{\star}-R^{k}\right\|_{L^{2}\left(\Gamma_{\text {out }}\right)}^{2}+\delta\right)^{2}
$$

i.e.

$$
\left\|u^{\prime}\left(R^{k}\right)\left(R^{k+1}-R^{k}\right)-\left(z^{\delta}-u\left(R^{k}\right)\right)\right\|_{L^{2}\left(\Gamma_{0}\right)} \leq c_{6}\left(\left\|R^{\star}-R^{k}\right\|_{L^{2}\left(\Gamma_{\text {out }}\right)}^{2}+\delta\right)
$$

where $c_{6}=\sqrt{3} \sqrt{\max \left\{c_{1}^{2}+L^{2} ; 1\right\}}$.

To prove (42), let us remark that by the definition of $\mathcal{J}\left(R^{k+1}\right)$, we have:

$$
\beta_{k}\left\|R^{k+1}-R^{k}\right\|_{L^{2}\left(\Gamma_{\text {out }}\right)}^{2} \leq \mathcal{J}\left(R^{k+1}\right) \leq \mathcal{J}\left(R^{\star}\right),
$$

which implies that:

$$
\left\|R^{k+1}-R^{k}\right\|_{L^{2}\left(\Gamma_{\text {out }}\right)}^{2} \leq \frac{1}{\beta_{k}} \mathcal{J}\left(R^{\star}\right)
$$

Using (43), we obtain:

$$
\left\|R^{k+1}-R^{k}\right\|_{L^{2}\left(\Gamma_{\text {out }}\right)}^{2} \leq \frac{1}{\beta_{k}}\left[2 c_{1}^{2}\left\|R^{\star}-R^{k}\right\|_{L^{2}\left(\Gamma_{\text {out }}\right)}^{4}+2 \delta^{2}\right]+\left\|R^{\star}-R^{k}\right\|_{L^{2}\left(\Gamma_{\text {out }}\right)}^{2}
$$

since before the end of iteration's process $\beta_{k}>\delta$ then $\frac{2 \delta^{2}}{\beta_{k}}<2 \delta$ and consequently:

$$
\left\|R^{k+1}-R^{k}\right\|_{L^{2}\left(\Gamma_{\text {out }}\right)}^{2} \leq\left[1+2 c_{1}^{2} \delta^{-1}\left\|R^{\star}-R^{k}\right\|_{L^{2}\left(\Gamma_{\text {out }}\right)}^{2}\right]\left\|R^{\star}-R^{k}\right\|_{L^{2}\left(\Gamma_{\text {out }}\right)}^{2}+2 \delta .
$$

As $R^{k} \in N\left(R^{\star}, b\right)$, we can majorate:

$$
\left\|R^{k+1}-R^{k}\right\|_{L^{2}\left(\Gamma_{\text {out }}\right)}^{2} \leq\left[1+2 c_{1}^{2} \delta^{-1} b^{2}\right]\left\|R^{\star}-R^{k}\right\|_{L^{2}\left(\Gamma_{\text {out }}\right)}^{2}+2 \delta
$$

so, we have:

$$
\left\|R^{k+1}-R^{k}\right\|_{L^{2}\left(\Gamma_{\text {out }}\right)}^{2} \leq c_{7}\left(\left\|R^{\star}-R^{k}\right\|_{L^{2}\left(\Gamma_{\text {out }}\right)}^{2}+\delta\right)
$$

where $c_{7}=\max \left\{1+2 c_{1}^{2} \delta^{-1} b^{2} ; 2\right\}$.

\section{Lemma 11}

Under the hypothesis (8), (35), (37), (39) and (40), if $R^{k}, R^{k+1}$ generated by (20) are in $N\left(R^{\star}, b\right)$, then, for $\delta$ sufficiently small, we have:

$$
\left\|R^{k+1}-R^{\star}\right\|_{L^{\infty}\left(\Gamma_{\text {out }}\right)} \leq c_{9}\left(\left\|R^{k}-R^{\star}\right\|_{L^{2}\left(\Gamma_{\text {out }}\right)}^{2}+\delta\right)
$$

where $c_{9}=\frac{1+c_{M}}{c_{5}}\left(c_{6}+c_{1} c_{7}+1\right), L$ and $c_{1}$ defined in Lemma 8, $c_{M}$ defined in (37) and $c_{7}$ in Lemma 10. 
Proof

On one hand, from (38) we have:

$$
\left\|R^{k+1}-R^{\star}\right\|_{L^{\infty}\left(\Gamma_{\text {out }}\right)} \leq \frac{1+c_{M}}{c_{5}}\left\|u\left(R^{k+1}\right)-u\left(R^{\star}\right)\right\|_{L^{2}(\Gamma)} .
$$

On the other hand, we have:

$$
\begin{aligned}
& \left\|u\left(R^{k+1}\right)-u\left(R^{\star}\right)\right\|_{L^{2}(\Gamma)} \leq\left\|u\left(R^{k+1}\right)-z^{\delta}\right\|_{L^{2}(\Gamma)}+\left\|z^{\delta}-u\left(R^{\star}\right)\right\|_{L^{2}(\Gamma)} \\
& \quad \leq\left\|u^{\prime}\left(R^{k}\right)\left(R^{k+1}-R^{k}\right)+u\left(R^{k}\right)-z^{\delta}-\left\{u^{\prime}\left(R^{k}\right)\left(R^{k+1}-R^{k}\right)+u\left(R^{k}\right)-u\left(R^{k+1}\right)\right\}\right\|_{L^{2}(\Gamma)}+\delta \\
& \quad \leq\left\|u^{\prime}\left(R^{k}\right)\left(R^{k+1}-R^{k}\right)+u\left(R^{k}\right)-z^{\delta}\right\|_{L^{2}(\Gamma)}+\left\|u^{\prime}\left(R^{k}\right)\left(R^{k+1}-R^{k}\right)+u\left(R^{k}\right)-u\left(R^{k+1}\right)\right\|_{L^{2}(\Gamma)}+\delta
\end{aligned}
$$

so using (41) and (23), we obtain:

$$
\left\|u\left(R^{k+1}\right)-u\left(R^{\star}\right)\right\|_{L^{2}(\Gamma)} \leq c_{6}\left(\left\|R^{k}-R^{\star}\right\|_{L^{2}\left(\Gamma_{\text {out }}\right)}^{2}+\delta\right)+c_{1}\left\|R^{k+1}-R^{k}\right\|_{L^{2}\left(\Gamma_{\text {out }}\right)}^{2}+\delta
$$

then (42) gives:

$$
\left\|u\left(R^{k+1}\right)-u\left(R^{\star}\right)\right\|_{L^{2}(\Gamma)} \leq c_{6}\left(\left\|R^{k}-R^{\star}\right\|_{L^{2}\left(\Gamma_{\text {out }}\right)}^{2}+\delta\right)+c_{1} c_{7}\left(\left\|R^{k}-R^{\star}\right\|_{L^{2}\left(\Gamma_{\text {out }}\right)}^{2}+\delta\right)+\delta
$$

or equivalently:

$$
\left\|u\left(R^{k+1}\right)-u\left(R^{\star}\right)\right\|_{L^{2}(\Gamma)} \leq\left(c_{6}+c_{1} c_{7}+1\right)\left(\left\|R^{k}-R^{\star}\right\|_{L^{2}\left(\Gamma_{\text {out }}\right)}^{2}+\delta\right) .
$$

Combining (45) and (46), we obtain:

$$
\left\|R^{k+1}-R^{\star}\right\|_{L^{\infty}\left(\Gamma_{\text {out }}\right)} \leq \frac{1+c_{M}}{c_{5}}\left(c_{6}+c_{1} c_{7}+1\right)\left(\left\|R^{k}-R^{\star}\right\|_{L^{2}\left(\Gamma_{\text {out }}\right)}^{2}+\delta\right)
$$

which is nothing but (44) with $c_{9}=\frac{1+c_{M}}{c_{5}}\left(c_{6}+c_{1} c_{7}+1\right)$.

\section{Remark 12}

Let us remark that by their definitions given in Lemma 10, we have $c_{6}=c_{6}(b) \geq \sqrt{3}$ and $c_{7}=c_{7}(b) \geq 2$.

For the sequel, let us note $c_{i}$ the constant of the inclusion of $L^{\infty}\left(\Gamma_{\text {out }}\right)$ into $L^{2}\left(\Gamma_{\text {out }}\right)$. We will assume, without loss of generality, that $c_{i}>1$. We also need to define, for a fixed $\bar{b} \in[0,1]$ and for $b, \delta \in[0,1]$, the following positive constants:

$$
\begin{aligned}
& \alpha(b)=\left[\sqrt{c_{7}(b)}+1\right] c_{9}(b) c_{i} \\
& \eta(b)=\alpha(b) \delta+\sqrt{c_{7}(b) \delta} \\
& r(b, \delta)=\min \left\{b ; \frac{b-\sqrt{c_{7}(\bar{b}) \delta}}{\sqrt{c_{7}(\bar{b})}+1}\right\}
\end{aligned}
$$

where $c_{9}$ is defined in the previous Lemma.

\section{Theorem 13}

Under the hypothesis of Theorem 4 and the hypothesis (8), (35), (37), (39) and (40), let $0<b<\bar{b}=\frac{1}{4}$ and let $r(b, \delta), \eta(\bar{b})$ and $\alpha(\bar{b})$ defined by $(47)$.

Suppose $\delta$ sufficiently small such that $r(b, \delta)>0$ and $1-4 \alpha(\bar{b}) \eta(\bar{b})>0$.

If we choose $b \in\left[\frac{1-\sqrt{1-4 \alpha(\bar{b}) \eta(\delta)}}{2 \alpha(\bar{b})} ; \frac{1+\sqrt{1-4 \alpha(\bar{b}) \eta(\delta)}}{2 \alpha(\bar{b})}\right]$ and if $R^{0} \in N\left(R^{\star}, r(b, \delta)\right)$, then the sequence $\left(R^{k}\right)$ generated by (20) is included in $N\left(R^{\star}, b\right)$ and satisfies:

$$
\left\|R^{k+1}-R^{\star}\right\|_{L^{2}\left(\Gamma_{\text {out }}\right)} \leq c_{i}\left\|R^{k+1}-R^{\star}\right\|_{L^{\infty}\left(\Gamma_{\text {out }}\right)} \leq c_{i} c_{9}(\bar{b})\left(\left\|R^{k}-R^{\star}\right\|_{L^{2}\left(\Gamma_{\text {out }}\right)}^{2}+\delta\right) .
$$


Proof

We will prove the result by induction in four steps.

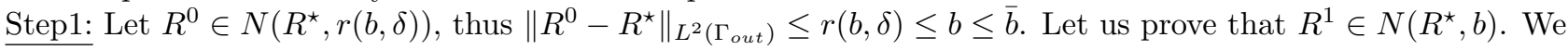
have:

$$
\left\|R^{1}-R^{\star}\right\|_{L^{2}\left(\Gamma_{\text {out }}\right)} \leq\left\|R^{1}-R^{0}\right\|_{L^{2}\left(\Gamma_{\text {out }}\right)}+\left\|R^{0}-R^{\star}\right\|_{L^{2}\left(\Gamma_{\text {out }}\right)} .
$$

Since $R^{0} \in N\left(R^{\star}, \bar{b}\right)$, we can apply (42) with $c_{7}=c_{7}(\bar{b})$ :

$$
\left\|R^{1}-R^{0}\right\|_{L^{2}\left(\Gamma_{\text {out }}\right)} \leq \sqrt{c_{7}(\bar{b})}\left(\left\|R^{0}-R^{\star}\right\|_{L^{2}\left(\Gamma_{\text {out }}\right)}+\sqrt{\delta}\right)
$$

therefore:

$$
\begin{aligned}
\left\|R^{1}-R^{\star}\right\|_{L^{2}\left(\Gamma_{\text {out }}\right)} & \leq \sqrt{c_{7}(\bar{b})}\left(\left\|R^{0}-R^{\star}\right\|_{L^{2}\left(\Gamma_{\text {out }}\right)}+\sqrt{\delta}\right)+\left\|R^{0}-R^{\star}\right\|_{L^{2}\left(\Gamma_{\text {out }}\right)} \\
& \leq\left[\sqrt{c_{7}(\bar{b})}+1\right]\left\|R^{0}-R^{\star}\right\|_{L^{2}\left(\Gamma_{\text {out }}\right)}+\sqrt{c_{7}(\bar{b})} \sqrt{\delta} \\
& \leq\left[\sqrt{c_{7}(\bar{b})}+1\right] r(b, \delta)+\sqrt{c_{7}(\bar{b}) \delta} \\
& \leq b
\end{aligned}
$$

since $r(b, \delta) \leq \frac{b-\sqrt{c_{7}(\bar{b}) \delta}}{\sqrt{c_{7}(\bar{b})}+1}$. Consequently, $R^{1} \in N\left(R^{\star}, b\right)$.

Step2: Suppose that $R^{1}, R^{2}, \cdots, R^{\ell} \in N\left(R^{\star}, b\right)$ and let us prove that $R^{\ell+1} \in N\left(R^{\star}, b\right)$. Since $R^{\ell} \in N\left(R^{\star}, b\right)$, we have from (42):

$$
\begin{aligned}
\left\|R^{\ell+1}-R^{\star}\right\|_{L^{2}\left(\Gamma_{\text {out }}\right)} & \leq\left\|R^{\ell+1}-R^{\ell}\right\|_{L^{2}\left(\Gamma_{\text {out }}\right)}+\left\|R^{\ell}-R^{\star}\right\|_{L^{2}\left(\Gamma_{\text {out }}\right)} \\
& \leq \sqrt{c_{7}(\bar{b})}\left[\left\|R^{\ell}-R^{\star}\right\|_{L^{2}\left(\Gamma_{\text {out }}\right)}+\sqrt{\delta}\right]+\left\|R^{\ell}-R^{\star}\right\|_{L^{2}\left(\Gamma_{\text {out }}\right)}
\end{aligned}
$$

i.e.:

$$
\left\|R^{\ell+1}-R^{\star}\right\|_{L^{2}\left(\Gamma_{\text {out }}\right)} \leq\left[\sqrt{c_{7}(\bar{b})}+1\right]\left\|R^{\ell}-R^{\star}\right\|_{L^{2}\left(\Gamma_{\text {out }}\right)}+\sqrt{c_{7}(\bar{b}) \delta} .
$$

On one hand and using the inclusion $L^{\infty}\left(\Gamma_{\text {out }}\right) \subset L^{2}\left(\Gamma_{\text {out }}\right)$, we have:

$$
\left\|R^{\ell}-R^{\star}\right\|_{L^{2}\left(\Gamma_{\text {out }}\right)} \leq c_{i}\left\|R^{\ell}-R^{\star}\right\|_{L^{\infty}\left(\Gamma_{\text {out }}\right)} .
$$

On the other hand and since $R^{\ell}, R^{\ell-1}, \cdots, R^{1} \in N\left(R^{\star}, b\right) \subset N\left(R^{\star}, \bar{b}\right)$, we can apply (44) with $\bar{b}$ :

$$
\left\|R^{\ell}-R^{\star}\right\|_{L^{\infty}\left(\Gamma_{\text {out }}\right)} \leq c_{9}(\bar{b})\left(\left\|R^{\ell-1}-R^{\star}\right\|_{L^{2}\left(\Gamma_{\text {out }}\right)}^{2}+\delta\right) .
$$

(49), (50) and (51) give then:

$$
\begin{aligned}
\left\|R^{\ell+1}-R^{\star}\right\|_{L^{2}\left(\Gamma_{\text {out }}\right)} & \leq\left[\sqrt{c_{7}(\bar{b})}+1\right] c_{9}(\bar{b}) c_{i}\left(\left\|R^{\ell-1}-R^{\star}\right\|_{L^{2}\left(\Gamma_{\text {out }}\right)}^{2}+\delta\right)+\sqrt{c_{7}(\bar{b}) \delta} \\
& \leq\left[\sqrt{c_{7}(\bar{b})}+1\right] c_{9}(\bar{b}) c_{i}\left(b^{2}+\delta\right)+\sqrt{c_{7}(\bar{b}) \delta}
\end{aligned}
$$

Using the definition of $\alpha(\bar{b})$, we can write:

$$
\left\|R^{\ell+1}-R^{\star}\right\|_{L^{2}\left(\Gamma_{\text {out }}\right)} \leq \alpha(\bar{b}) b^{2}+\alpha(\bar{b}) \delta+\sqrt{c_{7}(\bar{b}) \delta}=\alpha b^{2}+\eta
$$

where we have set for simplicity $\alpha=\alpha(\bar{b})$ and $\eta=\eta(\bar{b})$.

Step3: Let us set $E(b)=\alpha b^{2}-b+\eta$. Since $1-4 \alpha \eta>0$, the equation $E(b)=0$ have two solutions $b_{1}=$ 
$\frac{1-\sqrt{1-4 \alpha \eta}}{2 \alpha}$ and $b_{2}=\frac{1+\sqrt{1-4 \alpha \eta}}{2 \alpha}$.

On one hand, since $1-4 \alpha \eta<1$ thus $b_{1}>0$. On the other hand, since $c_{6} \geq \sqrt{3}$ and $c_{7} \geq 2$, we can deduce that $c_{9}>2$ and therefore $\alpha(\bar{b})=\left[\sqrt{c_{7}(\bar{b})}+1\right] c_{9}(\bar{b}) c_{i}>2(1+\sqrt{2}) c_{i}>4=\frac{1}{\bar{b}}$. Consequently $1+\sqrt{1-4 \alpha \eta}<2<2 \alpha \bar{b}$ which implies that $b_{2}<\bar{b}$.

A simple study allows us to conclude that for $b \in\left[b_{1} ; b_{2}\right], E(b) \leq 0$, i.e. $\alpha b^{2}+\eta \leq b$.

Step4: From Step3 and (52) we can finally conclude that $R^{\ell+1} \in N\left(R^{\star}, b\right)$ and consequently apply (44) to obtain:

$$
\left\|R^{\ell+1}-R^{\star}\right\|_{L^{\infty}\left(\Gamma_{\text {out }}\right)} \leq c_{9}(\bar{b})\left(\left\|R^{\ell}-R^{\star}\right\|_{L^{2}\left(\Gamma_{\text {out }}\right)}^{2}+\delta\right)
$$

using the inclusion of $L^{\infty}\left(\Gamma_{\text {out }}\right)$ in $L^{2}\left(\Gamma_{\text {out }}\right)$, (48) follows.

\section{Numerical Procedure: The Surrogate functional technique}

Recall that $\mathcal{J}\left(R^{k+1}\right)=\min _{R \in K} \mathcal{J}(R)$. Thus, $R^{k+1}$ must satisfy the optimality condition $\mathcal{J}^{\prime}\left(R^{k+1}\right) \xi=0$, for all $\xi \in L^{\infty}\left(\Gamma_{\text {out }}\right)$, where:

$$
\mathcal{J}^{\prime}(R) \xi=2\left\langle u^{\prime}\left(R^{k}\right)\left(R-R^{k}\right)-\left(z^{\delta}-u\left(R^{k}\right)\right) ; u^{\prime}\left(R^{k}\right) \xi\right\rangle_{L^{2}\left(\Gamma_{0}\right)}+2 \beta_{k}\left\langle R-R^{k} ; \xi\right\rangle_{L^{2}\left(\Gamma_{\text {out }}\right)} .
$$

which generates a difficult and expensive numerical methods. To simplify the minimization problem, we can apply the surrogate functional method $[10,22]$. This technique consists on adding a strictly positive term to $\mathcal{J}(R)$ so we still have a convex minimization but allows us to have an explicit expression of the minimizer.

Let us introduce the surrogate function:

$$
\begin{aligned}
\mathcal{J}^{s}\left(R, R^{k}\right)= & \mathcal{J}(R)+A\left\|R-R^{k}\right\|_{L^{2}\left(\Gamma_{\text {out }}\right)}^{2}-\left\|u^{\prime}\left(R^{k}\right)\left(R-R^{k}\right)-z^{\delta}\right\|_{L^{2}\left(\Gamma_{0}\right)}^{2} \\
= & \left\|u^{\prime}\left(R^{k}\right)\left(R-R^{k}\right)-\left(z^{\delta}-u\left(R^{k}\right)\right)\right\|_{L^{2}\left(\Gamma_{0}\right)}^{2}+\beta_{k}\left\|R-R^{k}\right\|_{L^{2}\left(\Gamma_{\text {out }}\right)}^{2} \\
& +A\left\|R-R^{k}\right\|_{L^{2}\left(\Gamma_{\text {out }}\right)}^{2}-\left\|u^{\prime}\left(R^{k}\right)\left(R-R^{k}\right)-z^{\delta}\right\|_{L^{2}\left(\Gamma_{0}\right)}^{2}
\end{aligned}
$$

where $A$ is a positive constant choosen such that $A\left\|R-R^{k}\right\|_{L^{2}\left(\Gamma_{\text {out }}\right)}^{2}-\left\|u^{\prime}\left(R^{k}\right)\left(R-R^{k}\right)-z^{\delta}\right\|_{L^{2}\left(\Gamma_{0}\right)}^{2} \geq 0$.

It's easy to verify that:

$$
\begin{gathered}
\mathcal{J}^{s}\left(R, R^{k}\right)=2\left\langle u^{\prime}\left(R^{k}\right)\left(R-R^{k}\right)-z^{\delta} ; u\left(R^{k}\right)\right\rangle_{L^{2}\left(\Gamma_{0}\right)}+\left\|u\left(R^{k}\right)\right\|_{L^{2}\left(\Gamma_{0}\right)}^{2}+\beta_{k}\left\|R-R^{k}\right\|_{L^{2}\left(\Gamma_{\text {out }}\right)}^{2}+A\left\|R-R^{k}\right\|_{L^{2}\left(\Gamma_{\text {out }}\right)}^{2} \\
=2\left\langle u^{\prime}\left(R^{k}\right)\left(R-R^{k}\right) ; u\left(R^{k}\right)\right\rangle_{L^{2}\left(\Gamma_{0}\right)}-2\left\langle z^{\delta} ; u\left(R^{k}\right)\right\rangle_{L^{2}\left(\Gamma_{0}\right)}+\left\|u\left(R^{k}\right)\right\|_{L^{2}\left(\Gamma_{0}\right)}^{2} \\
+\beta_{k}\left\|R-R^{k}\right\|_{L^{2}\left(\Gamma_{\text {out }}\right)}^{2}+A\left\|R-R^{k}\right\|_{L^{2}\left(\Gamma_{\text {out }}\right)}^{2}
\end{gathered}
$$

Using (12), we obtain:

$$
\begin{aligned}
\mathcal{J}^{s}\left(R, R^{k}\right)= & 2\left\langle u\left(R^{k}\right)\left(R-R^{k}\right) ; u^{\prime}\left(R^{k}\right)^{\star}\right\rangle_{L^{2}\left(\Gamma_{\text {out }}\right)}-2\left\langle z^{\delta} ; u\left(R^{k}\right)\right\rangle_{L^{2}\left(\Gamma_{0}\right)}+\left\|u\left(R^{k}\right)\right\|_{L^{2}\left(\Gamma_{0}\right)}^{2} \\
& +\beta_{k}\left\|R-R^{k}\right\|_{L^{2}\left(\Gamma_{\text {out }}\right)}^{2}+A\left\|R-R^{k}\right\|_{L^{2}\left(\Gamma_{\text {out }}\right)}^{2} \\
= & A\left\|R-R^{k}+\frac{1}{A} u\left(R^{k}\right) u^{\prime}\left(R^{k}\right)^{\star}\right\|_{L^{2}\left(\Gamma_{\text {out }}\right)}^{2}-A\left\|\frac{1}{A} u\left(R^{k}\right) u^{\prime}\left(R^{k}\right)^{\star}\right\|_{L^{2}\left(\Gamma_{\text {out }}\right)}^{2} \\
+ & \left\|u\left(R^{k}\right)\right\|_{L^{2}\left(\Gamma_{0}\right)}^{2}-2\left\langle z^{\delta} ; u\left(R^{k}\right)\right\rangle_{L^{2}\left(\Gamma_{0}\right)}+\beta_{k}\left\|R-R^{k}\right\|_{L^{2}\left(\Gamma_{\text {out }}\right)}^{2} \\
= & A\left\|R-R^{k}+\frac{1}{A} u\left(R^{k}\right) u^{\prime}\left(R^{k}\right)^{\star}\right\|_{L^{2}\left(\Gamma_{\text {out }}\right)}^{2}+\beta_{k}\left\|R-R^{k}\right\|_{L^{2}\left(\Gamma_{\text {out }}\right)}^{2}+T\left(R^{k}\right)
\end{aligned}
$$


where

$$
T\left(R^{k}\right)=\left\|u\left(R^{k}\right)\right\|_{L^{2}\left(\Gamma_{0}\right)}^{2}-2\left\langle z^{\delta} ; u\left(R^{k}\right)\right\rangle_{L^{2}\left(\Gamma_{0}\right)}-A\left\|\frac{1}{A} u\left(R^{k}\right) u^{\prime}\left(R^{k}\right)^{\star}\right\|_{L^{2}\left(\Gamma_{\text {out }}\right)}^{2} .
$$

Since $T\left(R^{k}\right)$ is independant of $R$, we thus have:

$$
\underset{R \in K}{\operatorname{argmin}} \mathcal{J}^{\mathcal{S}}\left(R, R^{k}\right)=\underset{R \in K}{\operatorname{argmin}} L(R)
$$

where:

$$
L(R)=A\left\|R-R^{k}+\frac{1}{A} u\left(R^{k}\right) u^{\prime}\left(R^{k}\right)^{\star}\right\|_{L^{2}\left(\Gamma_{\text {out }}\right)}^{2}+\beta_{k}\left\|R-R^{k}\right\|_{L^{2}\left(\Gamma_{\text {out }}\right)}^{2} .
$$

It's easy to verify that, for all $\xi \in L^{\infty}\left(\Gamma_{\text {out }}\right)$, we have:

$$
L^{\prime}(R) \xi=2\left\langle A\left(R-R^{k}+\frac{1}{A} u\left(R^{k}\right) u^{\prime}\left(R^{k}\right)^{\star}\right)+\beta_{k}\left(R-R^{k}\right) ; \xi\right\rangle_{L^{2}\left(\Gamma_{\text {out }}\right)}
$$

so, the optimality condition gives:

$$
R=R^{k}-\frac{1}{A+\beta_{k}} u\left(R^{k}\right) u^{\prime}\left(R^{k}\right)^{\star}
$$

This result allows us to state the following algorithm for the reconstruction of $R$ :

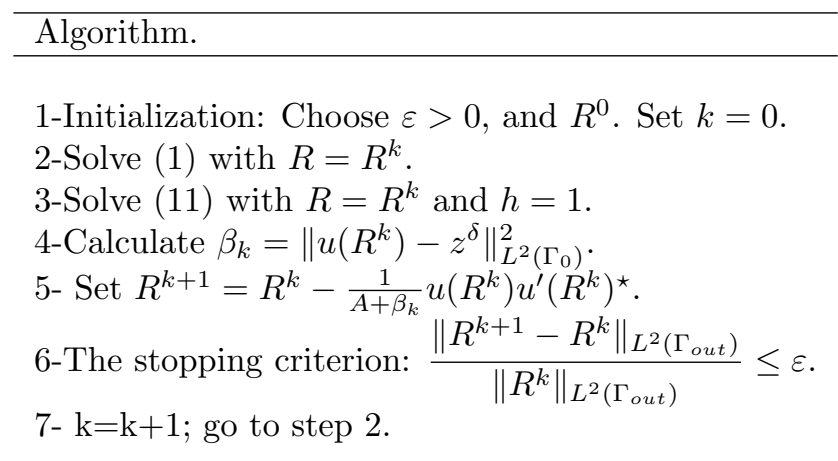

\section{$5 \quad$ Numerical Results}

To prove the efficiency of our study, we will appply the method with a two-dimensional annular domain $\Omega$ with radii $\rho_{1}=2$ and $\rho_{2}=1$. $\Gamma_{0}$ is a part of the circle with radii 2 corresponding to the angle $\theta \in[0,3 \pi / 2], \Gamma_{\ell}$ is te remainning part of this circle, while $\Gamma_{\text {out }}$ is the inner boundary i.e. the circle of radii 1 . We use the Freefem Software environnement [26]. The exact solution is generated synthetically using the basic solution of the Stokes problem:

$$
u(x, y)=\left(4 y^{3}-x^{2}, 4 x^{3}+2 x y-1\right), \quad p(x, y)=24 x y-2 x .
$$

The noisy data $z^{\delta}$ is obtained by using the following perturbations:

$$
z^{\delta}=u(1+\delta \omega) \text { on } \Gamma_{0}
$$

where $\delta$ is the amount of noise and $\omega$ is a random variable generated from a uniform distribution in $[-1,1]$. Tests are done for $\delta=0.02$ with different values of $N$, we have set $A=2, \varepsilon=0.01$ for $N=1,2$ and $\varepsilon=0.05$ 

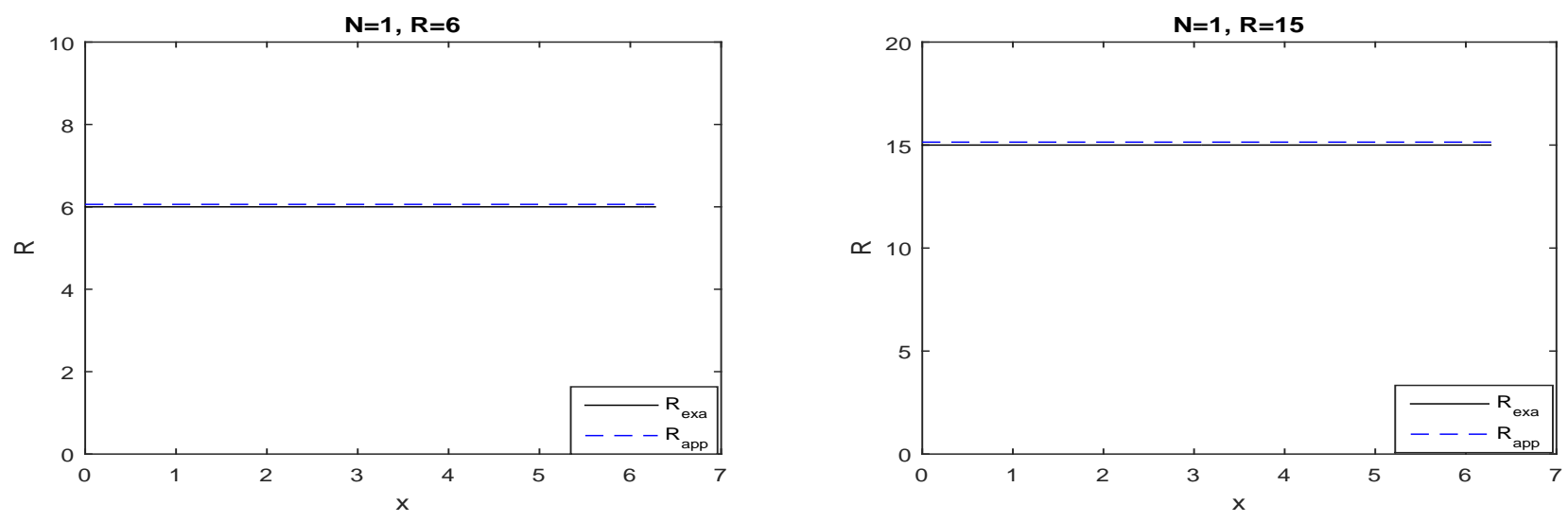

Figure 1: First test: $N=1 ; R=6$ (left) and $R=15$ (right)
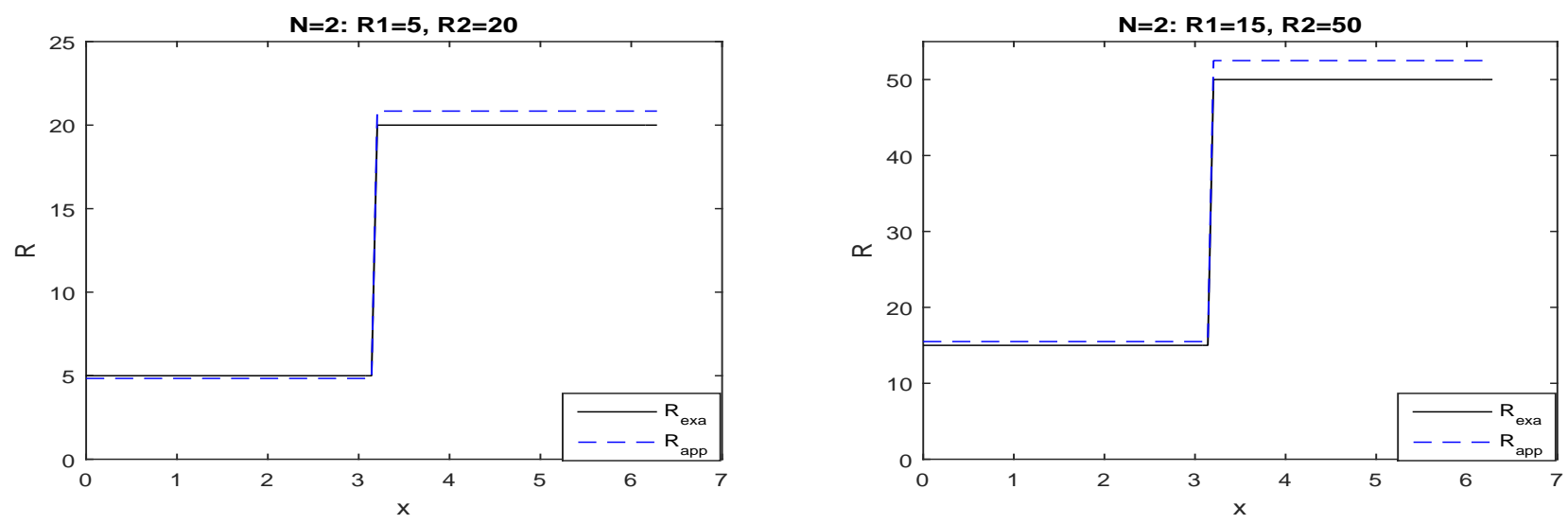

Figure 2: Second test: $N=2 ; R_{1}=5, R_{2}=20$ (left) and $R_{1}=15, R_{2}=50$ (right)

for $N=4$.

Figure 1 gives the result for the case $N=1$. We test with the Robin coefficient value $R_{1}=6$ (left) and $R_{1}=15$ (right). We have initialized the calculation with $R^{0}=10$ and $R^{0}=20$ respectively.

In figure 2 , we give the reconstruction when testing for $N=2$. First, we have tested with $\left(R_{1}, R_{2}\right)=(5,20)$, then with $\left(R_{1}, R_{2}\right)=(15,50)$. For the first case we choose $\left(R_{1}^{0}, R_{2}^{0}\right)=(8,24)$ as initial guesse and $\left(R_{1}^{0}, R_{2}^{0}\right)=(18,55)$ for the second one.

We finally test with $N=4$. The results are illustrated in Figure 3 . First for $\left(R_{1}, R_{2}, R_{3}, R_{4}\right)=(20,12,18,25)$ and then with $\left(R_{1}, R_{2}, R_{3}, R_{4}\right)=(30,55,8,40)$. The initial values are $\left(R_{1}^{0}, R_{2}^{0}, R_{3}^{0}, R_{4}^{0}\right)=(25,16,23,30)$ for the first case and $\left(R_{1}^{0}, R_{2}^{0}, R_{3}^{0}, R_{4}^{0}\right)=(35,61,12,45)$ for the second one.

All the results show that the method gives very good approximations of the true Robin coefficient values. The one bothering thing is that the initial values are poor ones which is the case for all Newton methods. The results are neverthless satisfying in view of the severe ill-posedness of the studied problem. Otherwise, in some cases, this may not be restrictive since the parameters to be identified can be roughrly estimated in the laboratory and these estimations can constitute a good initial values in order to obtain a precise identification. 

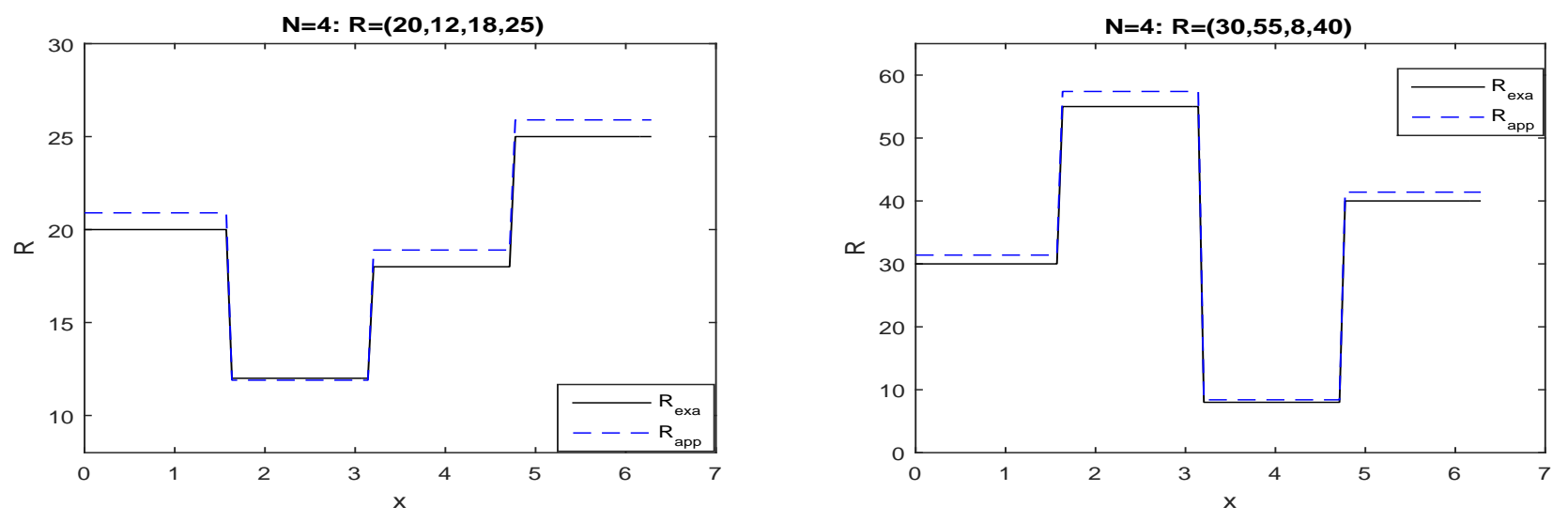

Figure 3: Third test: $N=4 ; R_{1}=20, R_{2}=12, R_{3}=18, R_{4}=25$ (left) and $R_{1}=30, R_{2}=55, R_{3}=8, R_{4}=40$ (right).

\section{Conclusion}

In this work, we have used the Levenberg-Marquardt method to solve the inverse problem of identifying a Robin coefficient corresponding to the Stokes system. This ill-posed problem is initially formulated as a nonlinear and nonconvex minimization. The LM method trasform it into a convex one. We prove the quadratic convergence of this method under the assumption that the Robin coefficient is piecewise constant on some part of the boundary on which the velocity of a given reference solution stays far from 0 . We have used the surrogate functional technique to simplify the numerical procedure and have proved the efficiency of our study by some numerical tests.

\section{References}

[1] L. Baffico, C. Grandmont and B. Maury, Multiscale modeling of the respiratory tract, Math. Models Methods Appl. Sci. 20 (2010), no. 1, 59-93.

[2] A. Ben Abda and F. Khayat, Reconstruction of missing boundary conditions from partially overspecified data: the Stokes system, ARIMA Journal 23 (2016), 79-100.

[3] D.P. Bertsekas, Nonlinear Programming, Belmont: Athena Sientific, 1995.

[4] M. Boulakia, A. C. Egloffe, and C. Grandmont, Stability estimates for a Robin coefficient in the twodimensional Stokes system, Mathematical Control and Related Field 3 (2013), 21-49.

[5] M. Boulakia, A. C. Egloffe, and C. Grandmont, Stability estimates for the unique continuation property of the Stokes system and for an inverse boundary coefficient problem, Inverse Problems 29 (2013) 115001.

[6] S. Chaabane, C. Elhechmi and M. Jaoua, A stable recovery method for the Robin inverse problem, Mathematics and Computers in Simulation 66 (2004), 367-383.

[7] S. Chaabane, J. Ferchichi and K. Kunisch, Differentiability properties of the L1-tracking functional and application to the Robin inverse problem, Inverse Problems 20 (2004), 1083-1097.

[8] S. Chaabane, I. Feki and N. Mars, Numerical reconstruction of a piecewise constant Robin parameter in the two- or three-dimensional case, Inverse Problems 28 (2012).

[9] Daijun Jiang and T. A. Talaat, Simultaneous identification of Robin coefficient and heat flux in an elliptic system, International Journal of Computer Mathematics 94 (2017), no. 1, 185-196. 
[10] I. Daubechies, M. Defrise and C. DeMol, An iterative thresholding algorithm for linear inverse problems, Comm. Pure Appl. Math. 57 (2004), no. 1, 1413-1457.

[11] A. C. Egloffe, Etude de quelques problèmes inverses pour le système de Stokes. Application aux poumons, PhD thesis Paris VI, 2012.

[12] A. C. Egloffe, Lipschitz stability estimate in the inverse Robin problem for the Stokes system, C. R. A. S. Paris (2013).

[13] A. C. Egloffe, Lipschitz stability estimate in the inverse Robin problem for the Stokes system, INRIA Research Report RR 8222 (2013).

[14] F. Facchinei, C. Kanzow, A nonsmooth inexat Newton method for the solution of large-scale nonlinear complementarity problems, Mathematical Programming 76 (1997), 493-512.

[15] W. Fang and M. Lu, A fast collocation method for an inverse boundary value problem, Int. J. Numer. Methods Eng. 59 (2004), 1563-1585.

[16] J.Y. Fan and Y.X. Yuan, On the Quadratic Convergence of the Levenberg-Marquardt Method without Nonsingularity Assumption, Computing 74 (2005), 23-39.

[17] D. Fasino and G. Inglese, An inverse Robin problem for Laplace's equation: theoretical results and numerical methods, Inverse Problems 15 (1999), 41-48.

[18] M.A. Fernandez, J.F. Gerbeau and V. Martin, Numerical simulation of blood flows through a porous interface, ESAIM: Mathematical Modelling and Numerical Analysis 42 (2008).

[19] H. P. Gavin, The Levenberg-Marquardt method for nonlinear least squares curve-fitting problems, Department of Civil and Environmental Engineering Duke University, 2013.

[20] M. Hanke, A regularization Levenberg-Marquardt scheme, with applications to inverse groundwater filtration problems, Inverse Problems 13 (1997), 79-95.

[21] B.T. Jin, Conjugate gradient method for the Robin inverse problem associated with the Laplace equation, Int. J. Numer. Methods Eng. 71 (2007), 433-453.

[22] K. Lange, D. R. Hunter and I. Yang, Optimization Transfer algorithms using surrogate objective functions, J. Comp. Graph. Stat. 9 (2000), 1-59.

[23] D.W. Marquardt, An algorithm for least-squares estimation of nonlinear parameters, Journal of the Society for Industrial and Applied Mathematics 11 (1963), no. 2, 431-441.

[24] B. Maury, The resistance of the respiratory system, from top to bottom, ESAIM: Proceedings and Surveys 47 (2014), 75-96.

[25] B. Maury, Méthode des éléments finis en élasticité, Ecole Polytechnique, 2007.

[26] O. Pironneau, F. Hecht, A. Hyaric and K. Ohtsuka, FreeFEM, URL: http://www. freefem. org (2006).

[27] A. Quarteroni and A. Veneziani, Analysis of a geometrical multiscale model based on the coupling of ODEs and PDEs for blood flow simulations, Multiscale Modeling Simul. 1 (2003), 173-95.

[28] A. Talaat, D. Xiaomao and C. Rongliang, A new method for simultaneously reconstructing the spacetime dependent Robin coefficient and heat flux in a parabolic system, International Journal of Numerical Analysis and Modeling 14 (2017), no. 6, 893-915.

[29] N. Yamashita and M. Fukushima, On the rate of convergence of the Levenberg-Marquardt method, Computing (Suppl. 15) (2001), 237-249. 
[30] N. Yamashita and M. Fukushima, The proximal point algorithm with genuine superlinear convergence for the monotone complementarity problem, SIAM Journal on Optimization 11 (2000), no. 2, 364-379.

[31] J. Zou, D. Jiang and H. Feng, Quadratic Convergence of Levenberg-Marquardt Method for Elliptic and Parabolic Inverse Robin Problems, ESAIM: M2AN DOI: https://doi.org/10.1051/m2an/2018016. 